\title{
RECENSIÓN
}

\section{EL PAÍS VASCO ANTE LAS DROGAS}

\author{
Amando Vega Fuente \\ Tercera Prensa-Hirugarren Prentsa SL 2000 \\ ISBN: 84-87303-57-9 \\ Número de páginas 300
}

Recensión por: Teresa Robledo de Dios. Subdirección General de Promoción de la Salud y Epidemiología. Ministerio de Sanidad y Consumo.

El «fenómeno de las drogas» está lleno de contradicciones y de tópicos tanto en su interpretación como en sus respuestas, ante él la educación se presenta no sólo como un recurso para acercarnos a su comprensión, sino también como un elemento imprescindible para la prevención y el tratamiento de sus consecuencias negativas (A. Vega, 2000). En este libro el objetivo de su autor es aproximarnos a la comprensión del proceso vivido en los últimos años y a la situación actual de la educación sobre las drogas en las escuelas vascas. Para ello, se ha realizado un intenso esfuerzo investigador, valorando los elementos de la acción educativa escolar.

Se trata de una aproximación a un proceso complejo, rico en compromiso y experiencias, con la pretensión de que sirva de apoyo a la respuesta educativa ante las necesidades planteadas por el fenómeno de las drogas.

El libro se estructura en tres partes, que abordan la cuestión de las drogas en el País Vasco, cuál ha sido y es la respuesta educativa escolar, para finalizar con una mirada hacia el futuro y hacia unas respuestas educativas coherentes, más adecuadas a las necesidades reales.

Revisaremos, a partir de aquí, con algo de detalle el contenido de cada una de las partes del libro.

La primera parte, tras revisar la literatura de la educación sobre las drogas en la escuela en el País Vasco, ofrece un examen tanto de la cuestión de las drogas en esta Comunidad, como del proceso seguido en la prevención y en la educación sobre drogas en los centros escolares.

En el capítulo 1 se analiza el fenómeno de las drogas en el País Vasco. Se presentan los diferentes abordajes de este complejo fenómeno; el consumo de unas sustancias denominadas drogas, con todas sus consecuencias individuales y sociales, no se puede comprender al margen de unos condicionantes históricos, culturales, económicos y políti$\cos$, donde la educación ha desempeñado y desempeña un papel importante. También se describen los principales resultados de diferentes estudios que analizan la realidad existente en el País Vasco, profundizando en el fenómeno del consumo juvenil de alcohol.

El capítulo 2, sobre la prevención en el País Vasco, estudia la acción preventiva que se ha venido desarrollando en esta Comunidad, desde sus inicios (no debemos olvidar que el País Vasco fue la primera Comunidad Autónoma que aprobó una ley específica sobre drogodependencias en 1978) hasta la nueva Ley Vasca de Drogodependencias, en 1998.

Los capítulos tercero y cuarto, tras justificar la acción educativa escolar y sus exigencias, presentan el Plan de Actuación para la 
Comunidad Autónoma Vasca y analizan los distintos recursos educativos (la familia, el sistema escolar y la comunidad).

Finalmente, una visión global de los programas preventivos en marcha más utilizados es dada en el capítulo 5.

La segunda parte, sobre la respuesta educativa escolar, presenta el estudio piloto sobre la percepción de los profesores y de otros profesionales relacionados con la educación escolar sobre las drogas, recogiendo tanto el proceso seguido como los resultados obtenidos en los diferentes aspectos contemplados en él: la prevención escolar, las tareas de la escuela, la coordinación con los servicios de la comunidad, la formación, etc. Con el contraste de perspectivas entre los diferentes profesionales, se pretende hacer una primera valoración de la situación de la acción educativa escolar sobre drogas dentro de los planes comunitarios asumidos en la Comunidad Autónoma Vasca. Se describe, detalladamente, la metodología de investigación seguida y los principales resultados obtenidos.
Las conclusiones del estudio aparecen en la tercera parte con una panorámica general de los resultados obtenidos con el trabajo realizado. Estas conclusiones, si, por una parte, se presentan como una síntesis de todo lo expuesto por otra, son consideraciones que abren nuevos horizontes para la reflexión y el estudio. Aunque los resultados encontrados, por cierto muy coincidentes con los resultados de otros estudios, no parecen muy optimistas por lo que a la práctica escolar se refiere (la «educación para la salud» como asignatura transversal no está asumida en más de la mitad de los centros estudiados..), hay que reconocer que mucho se ha progresado en la educación sobre las drogas, en presupuestos, en materiales, en equipos de trabajo, etc.

En definitiva, este libro nos ofrece una exhaustiva revisión de lo que ha sido y es la prevención de las drogodependencias en el País Vasco, y se constituye en un importante documento de referencia para todos aquellos profesionales que trabajamos en este campo, desde los diferentes ámbitos de actuación. 